\title{
Cognitive Vulnerability to Depression in Canadian and Chinese Adolescents
}

\author{
Randy P. Auerbach $\cdot$ Nicole K. Eberhart • \\ John R. Z. Abela
}

Published online: 11 August 2009

(C) The Author(s) 2009. This article is published with open access at Springerlink.com

\begin{abstract}
The goal of the current study was to compare diathesis-stress and transactional models of cognitive vulnerability to depression in samples of Canadian $(n=118)$ and Chinese $(n=405)$ adolescents. We utilized a six-month multi-wave, longitudinal design in order to examine whether (a) perceived control moderated the association between the occurrence of dependent interpersonal stressors and subsequent increases in depressive symptoms (i.e., a diathesis-stress perspective) and (b) dependent interpersonal stressors mediated the association between perceived control and subsequent increases in depressive symptoms (i.e., a transactional perspective). Results from idiographic, time-lagged, hierarchical linear modeling analyses indicated that for Canadian adolescents both diathesis-stress and transactional models were significant predictors of depressive symptomology. When examining the diathesis-stress model, boys, but not girls,
\end{abstract}

The research reported in this article was supported by a Canadian Psychiatric Research Foundation (CPRF) Award and a McGill University Sabbatic Leave Research Grant awarded to John R.Z. Abela, and a McGill University Social Sciences and Humanities Student Research Grant Awarded to Randy P. Auerbach.

R. P. Auerbach ( $\bowtie)$

Department of Psychology, McGill University,

Stewart Biological Sciences Building, 1205 Dr. Penfield Avenue,

Montreal, QC, Canada H3A 1B1

e-mail: randy.auerbach@mail.mcgill.ca

N. K. Eberhart

Department of Psychology, University of California,

Los Angeles, CA, USA

J. R. Z. Abela

Department of Psychology, Rutgers University,

Newark, NJ, USA who reported lower perceived control, reported higher levels of depressive symptoms following the occurrence of dependent interpersonal stress. Gender differences, however, were not present in the transactional model. In contrast, transactional, but not diathesis-stress, models were significant in Chinese adolescents, and gender differences did not emerge. Overall, these results may reflect culturally-relevant differences in the etiology of depression in Canadian and Chinese adolescents.

Keywords Depression - Cognitive vulnerability . Diathesis-stress $\cdot$ Stress generation $\cdot$ Adolescence $\cdot$ China

Adolescence is a critical developmental period marked by increased autonomy and maturation during which individuals explore new relationships and their identity. At the same time, adolescence is a period of drastic transition. Such a transition can be stressful, and as a result, symptoms of depression may be experienced (Hankin et al. 2007). The examination of subclinical depressive symptoms is important given that such symptoms are associated with an increased risk for future psychopathology (e.g., depressive, anxious, and behavioral disorders) as well as a wide range of negative outcomes including academic deficiencies, interpersonal difficulties with peers and family, and impaired cognitive functioning (Avenevoli et al. 2008; Kessler and Walters 1998; Reinherz et al. 1993). Subclinical levels of depressive symptoms are also associated with a number of poor outcomes in adulthood such as increased stressful life events, lower income levels, poorer social support, greater marital conflict, and higher incidence of substance use and dependence (Conway et al. 2006; Franko et al. 2005; Gotlib et al. 1998; Rao et al. 1995; Schepis and Rao 2005). 
One of the most robust concurrent and prospective predictors of depressive symptoms and episodes is the occurrence of stress (Grant et al. 2004; Shih et al. 2006). More specifically, Mazure (1998) found that approximately $50 \%$ of individuals diagnosed with depression experience severe stress prior to the onset of an episode. At the same time, research has also indicated that "even extreme stress is not linked to psychopathology in all individuals," as 50\% of individuals who experience stress do not develop psychopathology (Ingram and Luxton 2005, p. 32). Given that not all individuals who experience significant stress develop depression, recent prospective research has begun to utilize diathesis-stress models in order to examine the etiology of depression amongst adolescents (Ingram and Luxton 2005). Such models examine psychological vulnerabilities that are triggered following the occurrence of stressors that render certain adolescents susceptible to develop depression.

\section{Diathesis-Stress and Transactional Models of Depression}

To date, there has been a preponderance of research supporting cognitive vulnerability models of depression using a diathesis-stress framework amongst adolescent samples (for a review see Abela and Hankin 2008). Cognitive vulnerability models of depression examined within a diathesis-stress framework suggest that the occurrence of stress activates cognitive diatheses in vulnerable individuals (Beck 1983). Once activated, maladaptive cognitions then trigger a pattern of negatively biased, self-referent information processing that ultimately culminates in depression. Cognitive vulnerability theories posit that in the absence of stress, maladaptive cognitions do not arise, suggesting that such factors only contribute to depression in the presence of stress.

Recently, transactional models of depression have been receiving increased attention. While the diathesis-stress approach focuses on individuals' reactions to stressors, the transactional approach posits that individuals actively contribute to the occurrence of the stressors they experience, through a process labeled stress generation (Hammen 1991), which in turn contributes to depression. Self-generated stressors have alternatively been labeled dependent stressors because their occurrence is in part dependent on the actions of the individual, as opposed to fateful stressors that occur independent of a person's actions. While stress generation was initially examined in adults (e.g., Hammen 1991), there has been accumulating evidence for stress generation in children and adolescents (e.g., Adrian and Hammen 1993; Carter et al. 2006; Cole et al. 2006; Harkness et al. 2008; Rudolph et al. 2000; Rudolph 2008; Rudolph and Hammen 1999; Shih et al. 2009; Wingate and Joiner 2004).

\section{Cognitive Vulnerability}

Past research examining the relationship between cognitive vulnerability to depressive symptoms in adolescent samples have found strong support for a wide range of factors including attributional style (e.g., Abela and Hankin 2008), dysfunctional attitudes (e.g., Lewinsohn et al. 2001), and rumination (Abela et al. 2007). To date, less research has examined the role of low perceived control, the belief that one's actions do not exert influence over important life outcomes (Weisz et al. 2001). For individuals who maintain a perception that they have limited ability to change views of the self, world, and future (i.e., the negative cognitive triad) (Beck 1983), low perceived control may trigger helplessness and hopelessness cognitions which subsequently increase one's susceptibility to experience depressive symptomology. Past cross-sectional research has found that lower levels of perceived control are associated with higher levels of depressive symptoms (Margaro and Weisz 2006; Weisz et al. 1993). In contrast, main effect models examining the relationship prospectively have received inconsistent results (Muris et al. 2003; Weisz et al. 2001).

One possibility for these inconsistent prospective findings is that research has not examined perceived control within a diathesis-stress framework which may better examine the process with which a given cognitive vulnerability leads to depression. Inherent to diathesis-stress models is the belief that the interaction between pre-morbid vulnerability factors and situational stressors contributes to the onset of depressive symptomology (Monroe and Hadiyannakis 2002). Past research relying on either cross-sectional or main effect models makes the assumption that low levels of perceived control will uniformly and consistently predict higher levels of depressive symptoms without taking into account contextual and environmental factors such as stress (see Alloy et al. 1988). Given this methodological shortcoming, research is warranted to better understand the role that perceived control plays in the manifestation of depressive symptoms following the occurrence of stressors. As adolescents are particularly vulnerable to the deleterious effects of dependent interpersonal stress (Shih et al. 2006), we hypothesized that the interaction between such stress and perceived control would contribute to higher levels of depressive symptoms.

It may also be important to consider perceived control from a transactional perspective as relatively little research has examined cognitive predictors of stress generation and subsequent depression. Research in adolescent samples is particularly scarce, but preliminary evidence provides support for the role of cognitive vulnerability factors in transactional models via stress generation processes. In particular, a study of fifth and sixth graders found that negative social self-views and lower perceived control 
predicted stress generation in peer relationships (Caldwell et al. 2004), and another study of youth aged 6-14 found evidence that cognitive style, dependency, and selfcriticism predicted stress generation (Shih et al. 2009). However, it is unclear whether these results would replicate to a sample including older adolescents, and further, whether increased stress generation may mediate the relationship between these cognitive factors and depressed mood. To our knowledge, just one study has addressed this issue. In a sample of Israeli adolescents aged 14-16, Shahar and Priel (2003) provided evidence that negative life events mediated the effects of dependency and self-criticism on distress. This study represents an important step in applying a transactional perspective to understanding cognitive vulnerability to depression. At the same time, it should be noted that the study did not examine dependent stressors in particular, and additionally, the results need to be replicated using other cognitive variables including perceived control. Thus, we hypothesized that individuals with low perceived control who do not believe they have the capacity to control or influence their peer and familial relationships will experience increased levels of dependent interpersonal stress, and then such stress would contribute to higher levels of depressive symptoms.

\section{Secondary Objective: Examining Depressive Symptoms amongst Chinese Adolescents}

The primary objective of the manuscript was to examine diathesis-stress and transactional models of depression in a Canadian sample of adolescents. The secondary aim was to examine the cross-cultural generalizability and robustness of these findings in adolescents from mainland China. Research examining the prevalence of major depressive disorder amongst Chinese adolescents has indicated that rates are in line with Western samples (Fong 2006; Hankin et al. 2007; Liu et al. 1999; Zhenghua 2004). While there are drastic cultural differences between North America and China, the rapid modernization and urbanization throughout mainland China has resulted in younger generations embracing more Western values (Liu et al. 2000). These Western values have seemingly undercut traditional collectivistic ideals including the importance of interconnectedness within Chinese culture, the interdependent sense of self in which one's personal goals are subordinate to the collective aims, and the central role of the extended family (Auerbach et al. 2007; Yeh and Inose 2002; Yeh and Wang 2000). It has long been believed that these types of sociocultural factors have buffered Chinese individuals from experiencing depression (Lee et al. 2007), however, as China continues to experience social change, younger generations have begun to embrace more individualistic and materialistic values potentially increasing vulnerability to experience negative affect (Auerbach et al. 2009a, b). In light of this ideological metamorphosis, vulnerability models of depression developed in Western contexts have become increasingly relevant to Chinese adolescents.

To date, research has found that stress is a strong crosssectional and prospective predictor of depression in Chinese samples (e.g., Fong 2006; Liu et al. 2004), however, there is a paucity of research examining more complex, integrative models that delineate the mechanism through which stress leads to depression amongst Chinese adolescents. In one of the few studies examining prospective models of depression, Auerbach et al. $(2009 \mathrm{a}, \mathrm{b})$ found that Chinese adolescents who reported a greater tendency to utilize maladaptive as opposed to adaptive coping strategies moderated the relationship between higher levels of stress and subsequent depressive symptoms (i.e., a diathesis-stress perspective). At the same time, given culture-specific factors including the authoritative communist political structure and restricted educational opportunities (Fong 2006), it is likely that vulnerability factors such as a lack of perceived control regarding the self, world, and future underscores vulnerability to depression. More specifically, despite greater social freedoms as a result of increased personal wealth, individuals are unable to travel freely within and outside of mainland China. Additionally, while education provides a path to rise from poverty to prosperity, only 2.6 of the 9.5 million students who complete the university entrance exam will gain admittance (Fong 2006). Acute availability shortage at universities has fostered an environment in which adolescents experience an existence plagued by significant pressure on exams, increased peer competition, less free time, and high parental and teacher expectations (Liu et al. 2000). Such changes have resulted in an adolescent population that has less perceived control regarding their future, and preliminary cross-sectional research amongst Chinese adolescents has indicated that diminished control has contributed to a greater number of behavioral problems and higher rates of psychopathology (Liu et al. 2000). At the same time, prospective research is warranted to better understand the underlying role that perceived control plays in the development of psychopathology. In lieu of the ongoing cultural shift from collectivistic- to individualistic-oriented values, we hypothesized that similar results would be obtained for the diathesis-stress and transactional approaches when examining the Chinese as compared to the Canadian adolescents.

\section{Goals of the Current Study}

In order to address theoretical and empirical gaps in past research, the primary aim of the present study was to 
examine the role that perceived control played in both diathesis-stress and transactional models of depression in Canadian adolescents. In doing so, we examined whether: (a) perceived control strengthened the association between dependent interpersonal stress and depressive symptoms (i.e., diathesis-stress perspective) and (b) perceived control shaped the type of stressors that adolescents experience, which subsequently contributed to higher levels of depressive symptoms (i.e., transactional approach). Our secondary aim was to examine the cross-cultural applicability of these models in a sample of adolescents from mainland China. Further, as research has indicated that sex differences emerge during adolescence with girls experiencing both higher levels of depressive symptoms as well as a greater occurrence of depressive episodes (Hankin et al. 2007), we examined whether gender predicted differential outcomes in all models.

\section{Method}

\section{Participants}

Participants in the current study were recruited from high schools in Montreal, Canada and Yue Yang, China. The Canadian sample included 118 adolescents $(46 \%$ male and $54 \%$ female) between the ages of 12 and 18 (Mean $=15.17$; $\mathrm{SD}=1.22)$. The sample was $78.9 \%$ Caucasians, $5.6 \%$ Asians, 4.3\% African Americans, 2.5\% Native Americans, and $8.7 \%$ reported other as their ethnicity. Participants' predominant mother tongues were English $(83.2 \%)$ and French (13.7\%), however, 3.1\% of participants reported other.

Participants from mainland China were recruited from an urban high school in Yue Yang, Hunan. The final sample consisted of 405 high school students $(49.8 \%$ male and $50.2 \%$ females) whose ages ranged from 14 to 19 (mean = 16.18; $S D=0.95$ ). The sample was $97.1 \%$ Han and $3.9 \%$ ethnic minority. All participants reported that their mother tongue was Mandarin.

\section{Procedure}

Prior to the initial assessment (Time 1), letters of informed consent were sent home to parents describing the aims of the project and requesting consent for their child to participate. In the present study, all students who received parental consent also gave personal consent. During the initial assessment which occurred on school grounds, students completed a demographics form and the following questionnaires: (1) Center for Epidemiologic Studies Depression Scale (Radloff 1977), (2) Adolescent Life Event Questionnaire-Revised (Hankin and Abramson 2002), and
(3) the Perceived Control Scale for Children (Weisz et al. 1998). Follow-up assessments occurred every 6 weeks for Canadian adolescents (Times 2-4) and every month for Chinese adolescents (Times 2-7), and participants completed self-report measures assessing negative events and depressive symptoms.

While there were slight differences with respect to the length of time between assessments when examining Canadian and Chinese adolescents, assessments were spaced close enough together to enable participants to accurately recall events that had occurred within their life but far enough apart such that individuals would experience changes in their levels of stress and symptoms. With such research, 4-6 weeks has become the most commonly used time interval between the initial and follow-up assessments (see Abela and Hankin 2008). Additionally, as our dependent variable is the slope of the relationship between perceived control, dependent interpersonal stress, and depressive symptoms, multiple waves of observations enables a more reliable estimate of the slope.

\section{Measures}

The Chinese version of all self-report measures was developed using the back-translation method. First, the original version was translated into Chinese by one bilingual translator from the psychology department at Central South University (Changsha, Hunan). Next, the Chinese version was back-translated into English by another bilingual translator from the psychology department at McGill University. If discrepancies arose in the back-translation, translators worked cooperatively to make corrections to the Chinese version.

Center for Epidemiologic Studies Depression Scale (CES-D; Radloff 1977) The CES-D is a 20-item self-report measure that assesses levels of depressive symptoms. Examples of questions include: "I felt sad," "I felt hopeless about the future," and "I felt lonely." Items on the scale ranged from 0 to 3 with higher scores reflecting greater depressive symptomology. Participants reported how they felt during the past week by using the following scale: rarely $(<1$ day), some or a little of the time (1-2 days), occasionally or a moderate amount of time (3-4 days), and most or all of the time (5-7 days). Across administrations the Cronbach's alpha ranged from 0.91 to 0.94 for Canadian adolescents and 0.89 to 0.95 for Chinese adolescents which indicates high internal consistency.

Adolescent Life Events Questionnaire-Revised (ALEQ; Hankin and Abramson 2002) The ALEQ is a 57-item self-report questionnaire that was developed to assess a broad range of negative life events occurring in the past 
month. A consensus team consisting of three advanced doctoral students and one postdoctoral fellow rated whether each item was a) dependent (i.e., at least in part dependent on the actions of the individual) and b) interpersonal in nature. Items that were unanimously agreed upon were retained, and items in which disagreement arose were excluded. A total of 29 items were rated as both dependent and interpersonal, and thus were included in the present analyses. Examples of items are "You fought with your parents over your personal goals, desires, or choice of friends," and "You had an argument with a close friend." Participants were asked to indicate how often such events occurred on a Likert scale ranging from 0 (never) to 4 (always) with higher scores reflecting a greater number of negative life events. In the current study, Cronbach's alphas for the 29 items rated as dependent interpersonal stressors ranged from 0.88 to 0.90 for Canadian adolescents and 0.91 to 0.94 for Chinese adolescents across administrations indicating high internal consistency. Further, past research using the ALEQ with Chinese samples has indicated that it is associated with (a) higher levels of depressive symptoms, (b) greater risky behavior engagement, and (c) greater endorsement of maladaptive emotion regulation strategies (Auerbach et al. 2007, 2009a, b).

Perceived Control Scale (PCS; Weisz et al. 1998) The PCS is a 24-item self-report questionnaire measuring beliefs about one's perceived ability to exert control over outcomes in the academic, social, and behavioral domains, and as it is a trait measure, was assessed only at Time 1. Examples of questions include: "I can get good grades if I really try," "I can make friends with other kids if I really try," and "I cannot stay out of trouble no matter how hard I try." Participants are asked to rate items using a Likert scale ranging from 1 (very false) to 4 (very true) with higher scores reflecting a greater level of perceived control. The PCS has been shown across numerous studies to have strong test-retest reliability (e.g., Margaro and Weisz 2006). In the current study, the Cronbach's alpha was 0.88 for Canadian adolescents and 0.87 for Chinese adolescents indicating high internal consistency. Past research examining the validity of PCS in Chinese samples has indicated that it is positively associated with other measures of cognitive vulnerability including the children's dysfunctional attitudes scale as well as negatively correlated with levels of stress, anxiety, and depression (Auerbach et al. 2009a, b).

\section{Overview of Data Analytic Approach}

To test our hypothesis that individuals possessing lower levels of perceived control would report greater increases in depressive symptoms (Time $_{\text {T) }}$ following the occurrence of dependent interpersonal stressors (Time $_{\mathrm{T}-1)}$ than individuals possessing higher levels of perceived control (i.e., a diathesis-stress perspective), we utilized idiographic, time lagged, multilevel modeling. Analyses were completed using SAS (version 9.1) mixed procedure and maximum likelihood estimation. Our dependent variable was within-subject fluctuations in depressive $\operatorname{symptoms}_{(\text {Time }} \mathrm{T}$ ) during the follow-up interval. As follow-up depressive symptom(Time T) scores are a within-subject variable, such scores were centered at each participant's mean such that scores reflect upwards or downwards fluctuations in an individual's level of depressive symptoms compared to his or her mean level of symptoms. Our primary predictors of follow-up depressive symptom $_{(\text {Time T) }}$ scores were perceived control and fluctuations in dependent interpersonal stress (Time $_{\mathrm{T}-1)}$ during the follow-up interval. Prior to analyses, the perceived control scores, a between subject variable, were standardized. As follow-up dependent interpersonal stress (Time T-1) $_{\text {is }}$ a withinsubject predictor, scores were centered at each participant's mean prior to analyses such that scores reflect upwards or downwards fluctuations in an individual's reported occurrence of dependent interpersonal stress as compared to his or her mean level of stress.

In order to examine our proposed mediation models (i.e., transactional approach), we followed the guidelines set forth by Bauer et al. (2006). Such an approach is ideal for examining mediation models that include repeated measures, and given that the model is estimated in a single equation, one can directly estimate the covariance of the random effects that are encompassed in different Level 1 and Level 2 models. Consequently, Bauer et al. (2006) data analytic approach is preferable to mediation models that utilize a step-by-step process (e.g., Baron and Kenny 1986; Kenny et al. 2003). To examine whether dependent interpersonal stress $_{(\text {Time }}$ T-1) mediated the relationship between perceived control and elevations in depressive

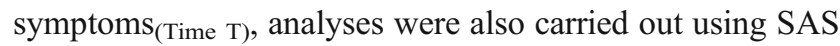
(version 9.1) mixed procedure and maximum likelihood estimation. Again, our dependent variable was withinsubject fluctuations in depressive symptoms (Time $_{\text {T) }}$ during the follow-up period . Our primary predictor of depressive symptoms (Time T) $_{\text {was }}$ werceived control, a between-subject predictor, and within-subject fluctuations of dependent interpersonal stress $_{(\text {Time T-1) }}$ was our mediator.

\section{Results}

\section{Descriptive Data}

Means, standard deviations, and intercorrelations between all Time 1 measures are included in Table 1 for Canadian 
Table 1 Pearson Correlations, Means, Standard Deviations, and Range for Depressive Symptoms, Dependent Interpersonal Stress, Perceived Control, and Age at the Initial Assessment for Canadian and Chinese Adolescents

\begin{tabular}{|c|c|c|c|c|c|c|c|c|}
\hline Variables & 1. & 2. & 3. & 4. & 5. & 6. & 7. & 8. \\
\hline \multicolumn{9}{|l|}{ Canadian Adolescents } \\
\hline 1. Initial Depressive Symptoms & - & & & & & & & \\
\hline 2. Initial Dependent Interpersonal Stress & $0.71^{* * *}$ & - & & & & & & \\
\hline 3. Perceived Control & $-0.48^{* * *}$ & $-0.62^{* * *}$ & - & & & & & \\
\hline 4. Age & 0.04 & 0.05 & -0.04 & - & & & & \\
\hline \multicolumn{9}{|l|}{ Chinese Adolescents } \\
\hline 5. Initial Depressive Symptoms & & & & & - & & & \\
\hline 6. Initial Dependent Interpersonal Stress & & & & & $0.56^{* * *}$ & - & & \\
\hline 7. Perceived Control & & & & & $-0.37^{* * *}$ & $-0.37^{* * *}$ & - & \\
\hline 8. Age & & & & & $0.20^{* * *}$ & $0.14^{* *}$ & 0.02 & - \\
\hline Mean & 13.84 & 24.93 & 37.38 & 15.17 & 12.16 & 24.86 & 43.77 & 16.18 \\
\hline Standard Deviation & 10.44 & 14.84 & 9.71 & 1.22 & 8.84 & 13.27 & 9.06 & 0.95 \\
\hline Low & 0 & 0 & 24 & 12 & 0 & 0 & 24 & 14 \\
\hline High & 47 & 72 & 65 & 18 & 44 & 77 & 74 & 19 \\
\hline
\end{tabular}

${ }^{*} p<0.05 ;{ }^{* *} p<0.01 ;{ }^{* * *} p<0.001$

and Chinese adolescents. Additionally, descriptive statistics for depressive symptoms during the follow-up period are presented in Table 2.

Canadian Adolescents: Idiographic, Time Lagged

Diathesis-Stress Models (Moderation)

In our first set of time lagged analyses, we were interested in examining the effects of the perceived control scores and dependent interpersonal stress (T-1) on individual's follow-up

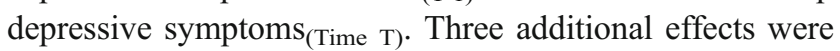
also included in this initial mean structure. First, in order to control for individual differences in baseline levels of depressive symptoms, participant's initial depressive symptom scores were included in the model. Second, in order to account for individual variability in the average level of depressive symptom scores at his/her mean level of dependent independent stress scores a random effect for intercept was included in the model. Last, given that follow-up dependent interpersonal $\operatorname{stress}_{(\mathrm{T}-1)}$ is a withinsubject predictor whose effect is expected to vary from participant to participant, a random effect for slope was included in the model.

The final results with respect to the fixed-effects component of the model are presented in Table 3. The first-order autoregressive covariance parameter was not significant $(r=-0.23, n s)$, however, it was retained in order to provide a more stringent examination of our hypothesis. The random intercept $(r=67.12, p<0.001)$ was significant and thus, retained in the model. The random slope was not significant, and consequently, it was removed from the model prior to re-estimation. Of primary importance, a significant two-way, cross-level interaction emerged between perceived control and follow-up dependent interpersonal stress ${ }_{(\mathrm{T}-1)}$. The fixed effects model was utilized to plot predicted follow-up depressive symptom scores(Time T) for

Table 2 Means, Standard Deviations, and Range for Depressive Symptoms during the Follow-up Period

\begin{tabular}{|c|c|c|c|c|c|c|c|c|}
\hline \multirow[b]{2}{*}{ Depressive Symptoms } & \multicolumn{4}{|c|}{ Canadian Adolescents } & \multicolumn{4}{|c|}{ Chinese Adolescents } \\
\hline & Mean & Standard Deviation & Low & High & Mean & Standard Deviation & Low & High \\
\hline Follow-Up \#1 & 12.95 & 10.26 & 0 & 48 & 12.36 & 9.22 & 0 & 51 \\
\hline Follow-Up \#2 & 11.37 & 10.99 & 0 & 51 & 12.02 & 9.53 & 0 & 51 \\
\hline Follow-Up \#3 & 9.96 & 10.57 & 0 & 50 & 11.35 & 9.70 & 0 & 58 \\
\hline Follow-Up \#4 & 12.83 & 11.10 & 0 & 46 & 10.32 & 9.99 & 0 & 51 \\
\hline Follow-Up \#5 & - & - & - & - & 8.96 & 9.10 & 0 & 51 \\
\hline Follow-Up \#6 & - & - & - & - & 10.00 & 9.56 & 0 & 54 \\
\hline
\end{tabular}

Depressive Symptoms = Center for Epidemiologic Studies Depression Scale (Radloff 1977) 
Table 3 Canadian Adolescents' Estimates of the Fixed Effects Component for the Time Lagged Model: Dependent Interpersonal Stress $($ Time T-1) $\times$ Perceived Control $\rightarrow$ Depressive Symptoms $($ Time T)

\begin{tabular}{|c|c|c|c|c|}
\hline Predictor & Parameter Estimate $(b)$ & Standard Error & $t$-Value & Degrees of Freedom (df) \\
\hline Age & 0.49 & 0.69 & 0.71 & 100 \\
\hline Gender & 3.31 & 1.79 & 1.85 & 100 \\
\hline Initial Depressive Symptoms & 5.82 & 0.97 & $5.97^{* * *}$ & 100 \\
\hline Dependent Interpersonal Stress $_{(\mathrm{T}-1)}$ & -0.06 & 0.05 & -1.11 & 115 \\
\hline Perceived Control & 1.00 & 0.93 & 1.07 & 100 \\
\hline Dependent Interpersonal Stress $_{(\mathrm{T}-1)} \times$ Perceived Control & 0.15 & 0.06 & $2.68^{* *}$ & 115 \\
\hline
\end{tabular}

${ }^{*} p<0.05 ;{ }^{* *} p<0.01 ;{ }^{* * *} p<0.001$

individuals with low or high perceived control scores (plus or minus 1.5 between-subject standard deviations) who reported a low or high occurrence of dependent interpersonal stress $_{(\text {Time }}$ T-1) (plus or minus 1.5 within-subject standard deviations) (see Fig. 1). Analyses were conducted for each perceived control condition examining whether the slope of the relationship between follow-up dependent interpersonal $\operatorname{stress}_{(\mathrm{T}-1)}$ and depressive symptoms $\left._{(\text {Time }} \mathrm{T}\right)$ significantly differed from 0 . Follow-up dependent interpersonal $\operatorname{stress}_{(\mathrm{T}-1)}$ was associated with increases in follow-up depressive symptoms (Time $T)_{\text {) }}$ for individuals possessing low levels of perceived control $(b=0.17, \mathrm{SE}=0.08, t(115)=$ $1.97, p<0.05)$, and it was associated with decreases in such symptoms for individual who reported high levels of perceived control $(b=-0.29, \mathrm{SE}=0.11, t(115)=-2.54$,

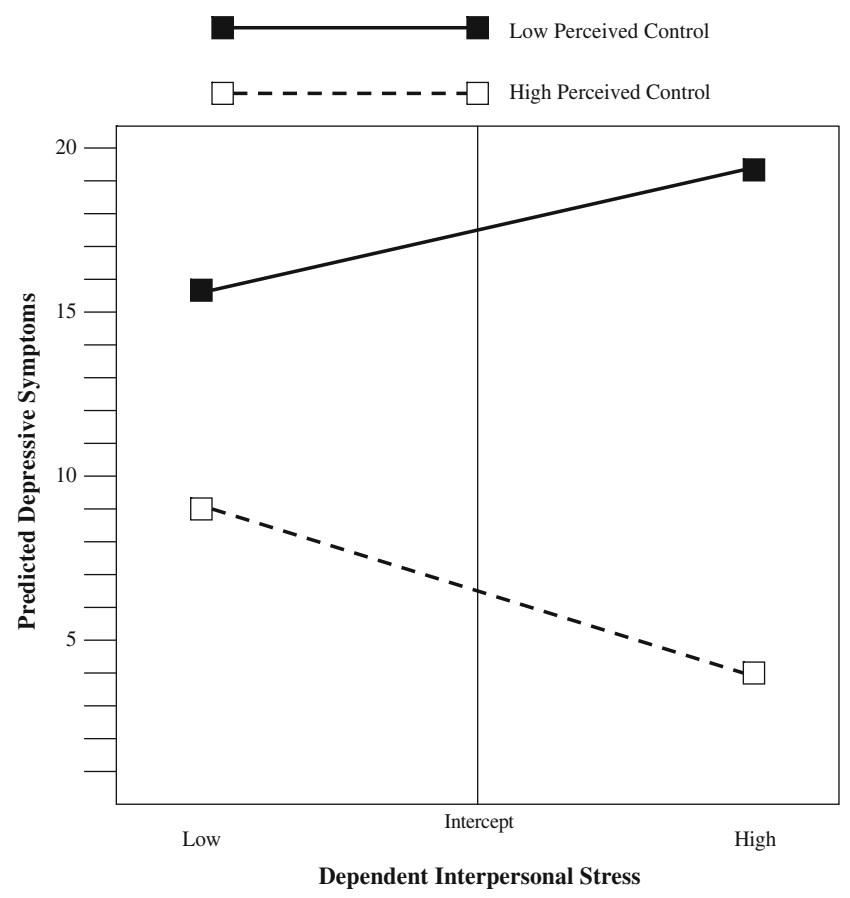

Fig. 1 Predicted depressive symptom scores as a function of perceived control and dependent interpersonal stress in Canadian adolescents $p<0.05)$. It is important to note that the slope of these two groups significantly differed $(b=0.45, \mathrm{SE}=0.17, t(115)=$ $2.68, p<0.01)$.

In order to determine whether gender was a moderator of the interaction between dependent interpersonal stress $_{(\mathrm{T}-1)}$ and perceived control, we examined a model that included dependent interpersonal $\operatorname{stress}_{(\mathrm{T}-1)}$, perceived control, and gender. Additionally, we included all two-way interactions and controlled for initial levels of depressive symptoms. Regarding random effects, the random intercept was not significant and thus removed prior to re-estimation. The first-order autoregressive covariance parameter $(r=0.84, p<$ $0.001)$ and random slope $(r=0.05, p<0.01)$ were significant and were thus retained in the model. When examining the fixed effects component of the model, a significant threeway, cross-level interaction emerged between dependent interpersonal stress $_{(\mathrm{T}-1)}$, perceived control, and gender (see Table 4). Analyses were conducted for boys and girls in each perceived control condition examining whether the slope of the relationship between dependent interpersonal $\operatorname{stress}_{(\text {Time T-1) }}$ and depressive symptoms $s_{(\text {Time T) }}$ significantly differed from 0. Among boys, the occurrence of dependent interpersonal stress (Time T-1) $_{\text {) }}$ was associated with increases in depressive symptoms (Time T) $_{\text {T) }}$ for individuals possessing low $(b=1.02, \mathrm{SE}=0.44, t(113)=2.31, p<0.05)$ but not high $(b=-0.95, \mathrm{SE}=0.62, t(113)=-1.52, n s)$ levels of perceived control. With regards to girls, the occurrence of dependent interpersonal stress (Time T-1) $_{\text {) }}$ was not significantly associated with increases in depressive symptoms $_{(\text {Time T) }}$ for individuals possessing low $(b=-0.29, \mathrm{SE}=0.25, t(113)=$ $-1.16, n s)$ or high $(b=0.35, \mathrm{SE}=0.29, t(113)=1.23, n s)$ levels of perceived control.

Canadian Adolescents: Idiographic, Transactional Model (Mediation)

Preliminary analyses indicated that none of the reported associations were moderated by either age or gender, and thus, analyses are presented for the entire sample as a whole. When examining the covariance structure, the best 
Table 4 Canadian Adolescents' Estimates of the Fixed Effects Component for the Time Lagged Model: Dependent Interpersonal Stress $($ Time T-1) $\times$ Perceived Control $\times$ Gender $\rightarrow$ Depressive Symptoms $($ Time T)

\begin{tabular}{|c|c|c|c|c|}
\hline Predictor & Parameter Estimate $(b)$ & Standard Error & $t$-Value & Degrees of Freedom (df) \\
\hline Age & 0.50 & 0.70 & 0.72 & 99 \\
\hline Gender & 3.42 & 1.79 & $1.90^{\#}$ & 99 \\
\hline Initial Depressive Symptoms & 5.86 & 0.97 & $6.03^{* * *}$ & 99 \\
\hline Dependent Interpersonal Stress $_{(\mathrm{T}-1)}$ & 0.04 & 0.12 & 0.29 & 113 \\
\hline Perceived Control & 0.41 & 1.27 & 0.32 & 99 \\
\hline Dependent Interpersonal Stress $_{(\mathrm{T}-1)} \times$ Perceived Control & 0.22 & 0.10 & $2.27^{\#}$ & 113 \\
\hline Dependent Interpersonal Stress $(\mathrm{T}-1) \times$ Gender & -0.002 & 0.15 & -0.01 & 113 \\
\hline Perceived Control $\times$ Gender & 1.20 & 1.70 & 0.71 & 99 \\
\hline Dependent Interpersonal Stress $(\mathrm{T}-1) \times$ Perceived Control $\times$ Gender & -0.29 & 0.14 & $-2.10^{*}$ & 113 \\
\hline
\end{tabular}

${ }^{\#} p<0.06 ;{ }^{*} p<0.05 ;{ }^{* *} p<0.01 ;{ }^{* * *} p<0.001$

fit was first-order autoregressive $(r=-0.26, p<.05)$. After choosing the covariance structure, we next examined the random-effects component of our model. Non-significant random-effects were deleted from the model prior to examining the fixed-effects component. The random intercept for dependent interpersonal stress $(r=41.16$, $p<0.001)$ was significant and thus, retained in the model. The random intercept for perceived control and the random slopes were not significant and were deleted from the model prior to re-estimation.

The final results with respect to the fixed-effects component of the model are presented in Table 5. Of primary importance, a significant mediation model emerged. More specifically, preliminary analyses indicated that lowers levels of perceived control predicted higher levels of depressive symptoms $(b=-3.51, \mathrm{SE}=0.91$, $t(114)=3.87, p<0.001)$. However, when controlling for the proportion of variance accounted for by dependent

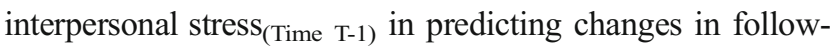

up depressive symptoms (Time T) $(b=0.18, \mathrm{SE}=0.03, t(542)=$ 5.42, $p<0.001$ ), high levels of dependent interpersonal $\left.\operatorname{stress}_{(\text {Time }} \mathrm{T}-1\right)$ fully mediated the relationship between low levels of perceived control and high levels of depressive symptoms $_{(\text {Time T) }}(b=0.20, \mathrm{SE}=0.75, t(542)=-0.27, n s)$.

Chinese Adolescents: Idiographic, Time Lagged DiathesisStress Models (Moderation)

In order to examine whether perceived control strengthened the association between dependent interpersonal stress $_{\text {(Time }}$ T-1) and depressive symptoms (Time T), we used the same data analytic approach outlined with the Canadian adolescents. Preliminary analyses indicated that none of the reported associations were moderated by either age or gender, and thus, analyses are presented for the sample as a whole. The first-order autoregressive parameter $(r=0.24, p<0.001)$, random intercept $(r=36.28, p<0.001)$, and random slope $(r=0.03, p<0.001)$ were significant and thus were retained

Table 5 Time Lagged Mediation Model for Canadian Adolescents: Estimates of the Fixed Effects Component

\begin{tabular}{|c|c|c|c|c|}
\hline Predictor & Parameter Estimate $(b)$ & Standard Error & $t$-Value & Degrees of Freedom (df) \\
\hline \multicolumn{5}{|l|}{ Dependent Interpersonal Stress: } \\
\hline Age & 0.16 & 0.75 & 0.22 & 542 \\
\hline Gender & 3.01 & 1.88 & 1.60 & 542 \\
\hline Initial Depressive Symptoms & 6.11 & 1.02 & $5.99^{* * *}$ & 542 \\
\hline Perceived Control & -4.58 & 1.01 & $4.53^{* * *}$ & 542 \\
\hline \multicolumn{5}{|l|}{ Depressive Symptoms $_{(\text {Time T) }}$ Model: } \\
\hline Age & 0.33 & 0.54 & 0.61 & 542 \\
\hline Gender & 2.50 & 1.37 & 1.82 & 542 \\
\hline Initial Depressive Symptoms & 4.54 & 0.77 & $5.86^{* * *}$ & 542 \\
\hline Dependent Interpersonal Stress ${ }_{(\text {Time }} \mathrm{T}-1$ ) & 0.19 & 0.03 & $5.42^{* * *}$ & 542 \\
\hline Perceived Control & 0.20 & 0.75 & -0.27 & 542 \\
\hline
\end{tabular}

${ }^{*} p<0.05,{ }^{* *} p<0.01,{ }^{* * *} p<0.001$ 
Table 6 Chinese Adolescents' Estimates of the Fixed Effects Component for the Time Lagged Model: Dependent Interpersonal Stress $($ Time T-1) $\times$ Perceived Control $\rightarrow$ Depressive Symptoms (Time T)

\begin{tabular}{|c|c|c|c|c|}
\hline Predictor & Parameter Estimate $(b)$ & Standard Error & $t$-Value & Degrees of Freedom (df) \\
\hline Age & 0.60 & 0.36 & 1.66 & 383 \\
\hline Gender & -1.03 & 0.68 & -1.53 & 383 \\
\hline Initial Depressive Symptoms & 5.31 & 0.37 & $14.34^{* * *}$ & 383 \\
\hline Dependent Interpersonal Stress $_{(\mathrm{T}-1)}$ & 0.07 & 0.02 & $3.51^{* * *}$ & 1428 \\
\hline Perceived Control & -0.66 & 0.36 & 1.81 & 383 \\
\hline 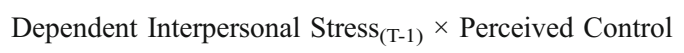 & -0.03 & 0.02 & -1.29 & 1428 \\
\hline
\end{tabular}

${ }^{*} p<0.05 ;{ }^{* *} p<0.01 ;{ }^{* * *} p<0.001$

in the model. The final results with respect to the fixedeffects component of the model are presented in Table 6. In contrast to the Canadian adolescents, a significant two-way, cross-level interaction did not emerge between perceived control and dependent interpersonal stress (Time T-1) $(b=0.03$, $\mathrm{SE}=0.02, t(1428)=-1.29, n s)$.

Chinese Adolescents: Idiographic, Transactional Model (Mediation)

As preliminary analyses indicated that the reported associations were not moderated by age or gender, results are presented for the entire sample. The first-order autoregressive covariance structure $(r=0.22, p<0.001)$, random intercept $(r=56.71, p<0.001)$, and random slope $(r=$ $30.40, p<0.001)$ were significant and retained in the model. The final results with respect to the fixed-effects component of the model are presented in Table 7. A significant mediation model emerged when examining the transactional model. Specifically, preliminary analyses indicated that lower levels of perceived control predicted higher levels of depressive symptoms $(b=-2.61, \mathrm{SE}=0.40, t(400)=6.65, p$ $<0.001$ ). Additionally, after controlling for the proportion of variance accounted for by dependent interpersonal stress (Time $_{\text {Tim }}$ T-1) in predicting changes in follow-up depressive symptom$\mathrm{S}_{\text {(Time T) }}(b=0.07, \mathrm{SE}=0.01 t(3237)=5.01, p<0.001)$, high levels of dependent interpersonal stress (Time $\mathrm{T}-1)$ fully mediated the relationship between low levels of perceived control and high levels of depressive symptoms (Time T) $(b=$ $0.46, \mathrm{SE}=0.34 t(3237)=1.37, n s)$.

\section{Discussion}

The present study examined the role of perceived control in diathesis-stress and transactional models of depression amongst Canadian and Chinese adolescents. Overall, the results suggest that both diathesis-stress and transactional models are applicable to Canadian adolescents. For Chinese adolescents, however, transactional, but not diathesis-stress models of perceived control, predict depressive symptomology. Such findings may reflect culturally-relevant differences in the etiology of depression.

When examining the diathesis-stress model amongst Canadian adolescents, results of time-lagged, idiographic analyses indicated that greater dependent interpersonal

Table 7 Time Lagged Mediation Model for Chinese Adolescents: Estimates of the Fixed Effects Component

\begin{tabular}{|c|c|c|c|c|}
\hline Predictor & Parameter Estimate $(b)$ & Standard Error & $t$-Value & Degrees of Freedom (df) \\
\hline \multicolumn{5}{|l|}{ Dependent Interpersonal Stress: } \\
\hline Age & 0.18 & 0.57 & 0.31 & 3237 \\
\hline Gender & -1.96 & 1.08 & -1.82 & 3237 \\
\hline Initial Depressive Symptoms & 4.87 & 0.59 & $8.23^{* * * *}$ & 3237 \\
\hline Perceived Control & -2.73 & 0.57 & $4.76^{* * *}$ & 3237 \\
\hline \multicolumn{5}{|l|}{ Depressive Symptoms $_{(\text {Time T) }}$ Model: } \\
\hline Age & 0.58 & 0.34 & 1.70 & 3237 \\
\hline Gender & -1.21 & 0.64 & -1.89 & 3237 \\
\hline Initial Depressive Symptoms $_{(\mathrm{T}-1)}$ & 4.95 & 0.36 & $13.80^{* * *}$ & 3237 \\
\hline Dependent Interpersonal Stress $($ Time T-1) & 0.07 & 0.01 & $5.01^{* * * *}$ & 3237 \\
\hline Perceived Control & -0.47 & 0.34 & 1.37 & 3237 \\
\hline
\end{tabular}

${ }^{*} p<0.05,{ }^{* *} p<0.01,{ }^{* * *} p<0.001$ 
stress interacted with low perceived control to predict increases in depressive symptoms. While children are prone to experience depressive symptoms as a direct consequence of negative events (Nolen-Hoeksema et al. 1992), Turner and Cole (1994) posit that adolescents' ability to utilize abstract reasoning increases the likelihood of developing stable cognitive vulnerabilities that are activated in the presence of stress. Thus, examining perceived control within a diathesis-stress framework becomes increasingly necessary amongst adolescents as such individuals are more likely to exhibit feelings of powerlessness in response to stressful life events which then leads to the onset of depressive symptoms. While these findings are in line with previous research examining the relationship between perceived control and depressive symptoms (e.g., Margaro and Weisz 2006; Weisz et al. 2001), the present study is an expansion in that it prospectively delineates the temporal relationship between perceived control, dependent interpersonal stress, and depressive symptoms.

Given that significant gender differences emerge in adolescence with regards to both level of depressive symptoms and number of depressive episodes (Hankin et al. 2007), we examined whether gender moderated the relationship between perceived control and dependent interpersonal stress to predict changes in depressive symptoms over time. Results indicated that boys, but not girls, who reported lower perceived control reported higher levels of depressive symptoms following the occurrence of dependent interpersonal stress. These findings are interesting to consider given that girls typically report greater depressive symptoms as compared to boys (Hankin et al. 2007), however, research examining gender-specific conflict resolution styles may provide a prism with which to better understand these results. In contrast to adolescent girls, boys who exhibit a diminished perception of control are more likely to act aggressively in response to interpersonal problems (Lindeman et al. 1997). The potentially violent response may be a physical manifestation of feeling powerless, and unfortunately, often exacerbates both the initial stressor and subsequent depressive symptoms (de Wied et al. 2007). With regards to girls who reported low levels of perceived control, the results suggest that the occurrence of interpersonal stress did not trigger or activate maladaptive responses that would serve to amplify interpersonal stress and depressive symptomology.

While diathesis-stress frameworks are a useful tool to understand cognitive vulnerability to depression, such models make the assumption that individuals are passive respondents to stress, with stress activating depressogenic perceptions in vulnerable individuals (e.g., Shih et al. 2009). In contrast, the transactional perspective asserts that individuals have the capacity to influence their environments by shaping the type of stressors they experience
(Hammen 1991). While Hammen's (1991) initial research on stress generation examined this phenomenon in adult women suffering from depression, more recent research has replicated these findings with adolescents (e.g., Harkness et al. 2008; Rudolph 2008). At the same time, less research with adolescent samples has examined whether cognitive vulnerability factors contribute to stress generation (for exceptions see Shahar and Priel 2003; Shih et al. 2009). The present study, to our knowledge, is the first to demonstrate that (a) low perceived control contributes to a greater occurrence of dependent interpersonal stress (i.e., stress generation) and (b) greater dependent interpersonal stress mediates the relationship between low perceived control and higher levels of depressive symptoms. Given that the model examines the time-lagged relationship between dependent interpersonal stress and depressive symptoms, it more clearly identifies the mechanism through which perceived control exerts its impact. In doing so, the results suggest that individuals who feel that they cannot exert an impact on important outcomes in their lives contribute to greater interpersonal conflict which in turn, results in higher levels of depressive symptoms.

With regards to the Chinese adolescents, in line with our hypothesis, the transactional model predicted significantly higher levels of depressive symptomology. In contrast to our hypothesis, the diathesis-stress model examining perceived control was not a predictor of fluctuations in such symptoms. These results may be the product of two sweeping changes that are occurring concurrently throughout mainland China. First, China's industrial growth has placed a heightened emphasis on education as it is viewed as a means to ensure future financial stability for both the individual and family. However, with less than $30 \%$ of students gaining admittance to university given the pervasive university shortage, it has resulted in (a) increased peer competition, (b) strict school discipline and obedience, (c) less recreational time, and (d) high expectations from teachers and parents (Liu et al. 2000). The intense pressure to succeed has likely diminished adolescents' beliefs that they control and shape the major decisions in their lives. Further, as approximately 7 million students do not gain admittance to the university despite committed and concerted efforts, many may feel that the process is hopeless. Second, historically, China has been a collectivistic society emphasizing the needs of the ingroup and fulfillment of social roles. However, China's industrial growth has resulted in younger generations embracing more individualistic and materialistic values (e.g., Auerbach et al. 2009a, b). Such a shift in values may be resulting in interpersonal tensions with parents and teachers as an individual's goal may no longer be subordinate to the collective aim. Taken together, the results from the present study indicate that feelings of diminished control lead individuals to contrib- 
ute, rather than react, to the occurrence of interpersonal stress, and thus, subsequently elevate levels of depressive symptomology.

While there are a number of strengths of the current study including the use of a multi-wave, longitudinal design and examining hypotheses with both Canadian and Chinese adolescents, it also important to recognize limitations. First, the current study utilized self-report measures to examine perceived control, stress, and depressive symptoms. While each of the measures utilized possesses strong reliability and validity, self-report measures are subject to a number of response biases. Given such a methodological shortcoming, future research would benefit from assessing cognitive vulnerability and symptoms with more sophisticated assessment techniques including semi-structured interviews, peer or parent ratings, and direct behavioral assessments. Second, while every effort was made to conduct parallel studies in Canada and China, scheduling difficulties emerged which resulted in a different number of waves as well as different time intervals between assessments. Third, the self-report measures utilized in our assessment of Chinese adolescents were translated from existing measures developed primarily for Western samples. Future research examining samples from mainland China would benefit from using and developing indigenous measures to assess both symptoms and personality characteristics. Last, the present study examined the relationship between perceived control and subsequent levels of dependent interpersonal stress, however, it did not assess independent stress. Thus, future research would benefit from determining whether perceived control was related to dependent as opposed to independent stress in order to provide a stronger examination of stress generation.

In sum, the present study examines theoreticallydriven models of cognitive vulnerability to depression in Canadian and Chinese adolescents. As differences between the Canadian and Chinese samples emerged when examining the role of perceived control, it suggests that the etiology of depression may vary. Whereas Chinese adolescents have a tendency to contribute to the manifestation of interpersonal stressors and subsequent depressive symptoms, Canadian adolescents seem to both generate and react to stressors in their lives. Ultimately, this may prove to have important clinical implications for the development of effective intervention and prevention programs as it provides greater insight into the development of depressive symptoms.

Open Access This article is distributed under the terms of the Creative Commons Attribution Noncommercial License which permits any noncommercial use, distribution, and reproduction in any medium, provided the original author(s) and source are credited.

\section{References}

Abela, J. R. Z., Aydin, C., \& Auerbach, R. P. (2007). Responses to depression in children: reconceptualizing the relation among response styles. Journal of Abnormal Child Psychology, 35, 913-927.

Abela, J. R. Z., \& Hankin, B. L. (2008). Depression in children and adolescents: Causes, treatment, and prevention. In J. R. Z. Abela \& B. L. Hankin (Eds.), Handbook of depression in children and adolescents (pp. 3-5). New York: Guilford.

Adrian, C., \& Hammen, C. (1993). Stress exposure and stress generation in children of depressed mothers. Journal of Consulting and Clinical Psychology, 61, 354-359.

Alloy, L. B., Hartlage, S., \& Abramson, L. Y. (1988). Testing the cognitive diathesis-stress theories of depression: Issues of research design, conceptualization, and assessment. In L. Alloy (Ed.), Cognitive processes in depression (pp. 31-73). New York: Guildford.

Auerbach, R. P., Abela, J. R. Z., \& Zhu, X. (2009). Examining the reliability and predictive validity of cognitive vulnerability measures in a sample of Chinese adolescents and university students (Submitted).

Auerbach, R. P., Abela, J. R. Z., Zhu, X., \& Yao, S. (2009). Understanding the role of coping in the development of depressive symptoms: symptom specificity, gender differences, and cross-cultural applicability (Revision Submitted).

Auerbach, R. P., Abela, J. R. Z., Zhu, X., \& Yao, S. (2007). A Diathesisstress model of engagement in risky behaviors in Chinese adolescents. Behaviour Research and Therapy, 45, 2850-2860.

Auerbach, R. P., McWhinnie, C. M., Goldfinger, M., Abela, J. R. Z., Zhu, X., \& Yao, S. (2009). The cost of materialism in a collectivistic culture: predicting risky behavior engagement in Chinese adolescents. Journal of Clinical Child and Adolescent Psychology.

Avenevoli, S., Knight, E., Kessler, R. C., \& Merikangas, K. R. (2008). Epidemiology of depression in children and adolescents. In J. R. Z. Abela \& B. L. Hankin (Eds.), Handbook of depression in children and adolescents (pp. 6-32). New York: Guilford.

Baron, R. M., \& Kenny, D. A. (1986). The moderator-mediator variable distinction in social psychological research: conceptual, strategic, and statistical considerations. Journal of Personality and Social Psychology, 51, 1173-1182.

Bauer, D. J., Preacher, K. J., \& Gil, K. M. (2006). Conceptualizing and testing random indirect effects and moderated mediation in multilevel models: new procedures and recommendations. Psychological Methods, 11, 142-163.

Beck, A. T. (1983). Cognitive therapy of depression: New perspectives. In P. J. Clayton \& J. E. Barrett (Eds.), Treatment of depression: Old controversies and new approaches (pp. 265290). New York: Raven.

Caldwell, M. S., Rudolph, K. D., Troop-Gordon, W., \& Kim, D.-Y. (2004). Recipricol influences among relational self-views, social disengagement, and peer stress during early adolescence. Child Development, 75, 1140-1154.

Carter, J. S., Garber, J., Ciesla, J. A., \& Cole, D. A. (2006). Modeling relations between hassles and internalizing symptoms in adolescents: a four-year prospective study. Journal of Abnormal Psychology, 115, 428-442.

Cole, D. A., Nolen-Hoeksema, S., Girgus, J., \& Gilda, P. (2006). Stress exposure and stress generation in child and adolescent depression: a latent trait-state-error approach to longitudinal analyses. Journal of Abnormal Psychology, 115, 40-51.

Conway, K. P., Compton, W., Stinson, F. S., \& Grant, B. F. (2006). Lifetime comorbidity of DSM-IV mood and anxiety disorders and specific drug use disorders: resutls form the national epidemiologic survey on alcohol and related conditions. Journal of Clinical Psychiatry, 67, 247-257. 
de Wied, M., Branje, S. J. T., \& Meeus, W. H. J. (2007). Empathy and conflict resolution in friendship relations among adolescents. Aggressive Behavior, 33, 48-55.

Fong, V. L. (2006). Coming of age under China's one-child policy. Stanford: Stanford University Press.

Franko, D. L., Striegel-Moore, R. H., Brown, K. M., Barton, B. A., McMahon, R. P., Schreiber, G. B., et al. (2005). Expanding our understanding of the relationship between negative life events and depressive symptoms in black and white adolescent girls. Psychological Medicine, 34, 1319-1329.

Gotlib, I. H., Lewinsohn, P. M., \& Seeley, J. R. (1998). Consequences of depression during adolescence: marital status and marital functioning in early adulthood. Journal of Abnormal Psychology, 107, 686-690.

Grant, K. E., Compas, B. E., Thurm, A. E., McMahon, S. D., \& Gipson, P. Y. (2004). Stressors and child and adolescent psychopathology: measurement issues and prospective effects. Journal of Clinical Child and Adolescent Psychology, 33, 412-425.

Hammen, C. (1991). Generation of stress in the course of unipolar depression. Journal of Abnormal Psychology, 100, 555-561.

Hankin, B. L., \& Abramson, L. Y. (2002). Measuring cognitive vulnerability to depression in adolescence: reliability, validity, and gender differences. Journal of Clinical Child and Adolescent Psychology, 31, 491-504.

Hankin, B. L., Mermelstein, R., \& Roesch, L. (2007). Sex differences in adolescent depression: stress exposure and reactivity models. Child Development, 78, 279-295.

Harkness, K. L., Lumley, M. N., \& Truss, A. E. (2008). Stress generation in adolescent depression: the moderating role of child abuse and neglect. Journal of Abnormal Child Psychology, 36, 421-432.

Ingram, R. E., \& Luxton, D. D. (2005). Vulnerability-stress models. In B. L. Hankin \& J. R. Z. Abela (Eds.), Development of psychopathology (pp. 32-46). New York: Guilford.

Kenny, D. A., Korchmaros, J. D., \& Bolger, N. (2003). Lower level mediation in multilevel models. Psychological Methods, 8, 115-128.

Kessler, R. C., \& Walters, E. E. (1998). Epidemiology of DSM-III-R major depression and minor depression among adolescents and young adults in the National Comorbidity Survey. Depression and Anxiety, 7, 3-14.

Lee, S., Tsang, A., \& Kwok, K. (2007). Twelve-month prevalence, correlates, and treatment preference of adults with DSM-IV major depressive episode in Hong Kong. Journal of Affective Disorders, 98, 129-136.

Lewinsohn, P. M., Joiner, T. E., \& Rohde, P. (2001). Evaluation of cognitive diathesis-stress models in predicting major depressive disorder in adolescents. Journal of Abnormal Psychology, 110, 203-215.

Lindeman, M., Harakka, T., \& Keltikangas-Järvinen, L. (1997). Age and gender differences in adolescents' reactions to conflict situations: aggression, prosociality, and withdrawal. Journal of Youth and Adolescence, 26, 339-351.

Liu, X., Kurita, H., Uchiyama, M., Okawa, M., Liu, L., \& Ma, D. (2000). Life events, locus of control, and behavioral problems among Chinese adolescents. Journal of Clinical Psychology, 56, $1565-1577$.

Liu, X., Sun, L., Liu, L., Yang, J., Li, C., Zhao, G., et al. (1999). Selfreported depression and its correlates in adolescents from a disaster area. Chinese Journal of Clinical Psychology, 7, 24-27.

Liu, R. X., Tein, J. Y., \& Zhao, Z. (2004). Coping strategies and behavioral/emotional problems among Chinese adolescents. Psychiatry Research, 126, 275-285.

Margaro, M. M., \& Weisz, J. R. (2006). Perceived control mediates the relation between parental rejection and youth depression. Journal of Abnormal Child Psychology, 34, 867-876.

Mazure, C. (1998). Life stressors as risk factors in depression. Clinical Psychology: Science and Practice, 5, 291-313.
Monroe, S. M., \& Hadiyannakis, H. (2002). The social environment and depression: Focusing on severe life stress. In I. H. Gotlib \& C. L. Hammen (Eds.), Handbook of depression (pp. 314-340). New York: Guilford.

Muris, P., Schouten, E., Meesters, C., \& Gijsbers, H. (2003). Contigency-Competence-Control Related beliefs and symptoms of anxiety and depression in a young adolescent sample. Child Psychiatry and Human Development, 33, 325-339.

Nolen-Hoeksema, S., Girgus, J. S., \& Seligman, M. P. (1992). Predictors and consequences of childhood depressive symptoms: a 5-year longitudinal study. Journal of Abnormal Psychology, $101,405-422$.

Radloff, L. S. (1977). The CES-D Scale: a self-report depression scale for research in the general population. Applied Psychological Measurement, 1, 385-401.

Rao, U., Ryan, N. D., Birmaher, B., Dahl, R. E., Wiliamson, D. R., Kaufman, J., et al. (1995). Unipolar depression in adolescents: clinical outcome in adulthooD. Journal of the American Academy of Child and Adolescent Psychiatry, 34, 566-578.

Reinherz, H. Z., Giaconia, R. M., Pakiz, B., Silverman, A. B., Frost, A. K., \& Lefkowitz, E. S. (1993). Psychosocial risks for major depression in late adolescence: a longitudinal community study. Journal of the American Academy of Child and Adolescent Psychiatry, 32, 1155-1163.

Rudolph, K. D. (2008). Developmental influences on interpersonal stress generation in depressed youth. Journal of Abnormal Psychology, 117, 673-679.

Rudolph, K. D., \& Hammen, C. (1999). Age and gender as determinants of stress exposure, generation, and reactions in youngsters: a transactional perspective. Child Development, 70, 660-677.

Rudolph, K. D., Hammen, C., Burge, D., Lindberg, N., Harzberg, D., \& Daley, S. E. (2000). Development and Psychopathology, 12, 215-234.

Schepis, T. S., \& Rao, U. (2005). Epidemiology and etiology of adolescent smoking. Current Opinion in Pediatrics, 17, 607-612.

Shahar, G., \& Priel, B. (2003). Active vulnerability, adolescent distress, and the mediating/suppressing role of life events. Personality and Individual Differences, 35, 199-218.

Shih, J. H., Abela, J. R. Z., \& Starrs, C. (2009). Cognitive and interpersonal predictors of stress generation in children of affectively ill parents. Journal of Abnormal Child Psychology.

Shih, J. H., Eberhart, N. K., Hammen, C. L., \& Brennan, P. A. (2006). Differential exposure and reactivity to interpersonal stress predict sex differences in adolescent depression. Journal of Clinical Child and Adolescent Psychology, 35, 103-115.

Turner, J. E., \& Cole, D. A. (1994). Developmental differences in cognitive diatheses in child depression. Journal of Abnormal Child Psychology, 22, 15-32.

Weisz, J. R., Sweeney, L., Proffitt, V., \& Carr, T. (1993). Controlrelated beliefs and self-reported depressive symptoms in late childhood. Journal of Abnormal Psychology, 102, 411-418.

Weisz, J. R., Southam-Gerow, M. A., \& McCarty, C. A. (2001). Controlrelated beliefs and depressive symptoms in clinic-referred children and adolescents: developmental differences and model specificity. Journal of Abnormal Psychology, 110, 97-109.

Weisz, J. R., Southam-Gerow, M. A., \& Sweeney, L. (1998). The perceived control scale for children. Los Angeles: University of California.

Wingate, L. R., \& Joiner, T. E. (2004). Depression-related stress generation: a longitudinal study of Black adolescents. Behavior Therapy, 35, 247-261.

Yeh, C. J., \& Inose, M. (2002). Difficulties and coping strategies of Chinese, Japanese and Korean immigrant students. Adolescence, 37, 69-82.

Yeh, C. J., \& Wang, Y. W. (2000). Asian American coping attitudes, sources, and practice: implications for indigenous counseling strategies. Journal of College Student Development, 41, 94-103.

Zhenghua, W. (2004). Minors suffer from anxiety, depression. China Daily. 